\begin{tabular}{|c|c|}
\hline Title & Photoswitchable Faraday effect in EuS-A u nanosystems \\
\hline Author(s) & Kawashima, A kira; Nakanishi, Takayuki; Kitagawa, Y uichi; Fushimi, Koji; Hasegawa, Y asuchika \\
\hline Citation & $\begin{array}{l}\text { Physica Status Solidi A applications and material s science, 213(1), 178-182 } \\
\text { https://doi.org/10.1002/pssa.201532526 }\end{array}$ \\
\hline Issue Date & 2016-01 \\
\hline Doc URL & http://hdl.handle.net/2115/64420 \\
\hline Rights & $\begin{array}{l}\text { This is the peer reviewed version of the following article: Physica Status Solidi A applications and materials science } \\
\text { V olume } 213 \text {, Issue 1, pages 178-182, January } 2016 \text {, which has been publi ished in final form at } \\
\text { http://doi.org/10.1002/pssa.201532526. This article may be used for non-commercial purposes in accordance with } \\
\text { Wiley Terms and Conditions for Self-A rchiving. }\end{array}$ \\
\hline Tyре & article (author version) \\
\hline File Information & Hasegawa PSSA 213(1).pdf \\
\hline
\end{tabular}

Instructions for use 


\title{
Photo-switchable Faraday Effect in EuS-Au Nanosystems
}

\author{
Akira Kawashima, Takayuki Nakanishi, Yuichi Kitagawa, Koji Fushimi, and Yasuchika Hasegawa *
}

Division of Applied Chemistry, Faculty of Engineering, Hokkaido University, North-13 West-8, Kita-ku, 060-8628 Sapporo, Japan

Received ZZZ, revised ZZZ, accepted ZZZ

Published online ZZZ (Dates will be provided by the publisher.)

Keywords Europium, Gold, Nanoparticles, Magnetic circular dichroism

* Corresponding author: e-mail hasegaway@eng.hokudai.ac.jp, Phone: +81 11706 7114, Fax: +81 117067114

\begin{abstract}
Effective photo-switchable europium sulfide nanocrystals with gold nanoparticles using dithiol (DDT: 1,10-decanedithiol) joint molecules, EuS-Au nanosystems, are demonstrated. The TEM image indicates the formation of EuS-Au nanosystems composed of cube-shaped EuS nanocrystals and spherical $\mathrm{Au}$ nanoparticles. Under visible light irradiation, drastic change of absorption band of EuS-Au nanosystems at around $600 \mathrm{~nm}$ was observed. The Faraday effects of EuS-Au nanosystems were estimated using magnetic circular dichroism (MCD) measurements. The effective change of the MCD spectra of EuS-Au nanosystems under visible light irradiation was successfully observed at around $670 \mathrm{~nm}$ for the first time. The effective reversible changes in MCD spectra with the alternative irradiation cycles of visible light ( $>440 \mathrm{~nm}$ ) and dark are also presented. The decrease rate of rotation angle at 670 $\mathrm{nm}$ of EuS-Au nanosystems is larger than that of absorbance. These results indicate that the effective change of MCD spectra of EuS-Au nanosystems would be dominated not only by drastic change of absorption
\end{abstract}

band related to enhanced LSPR of Au nanoparticles but also by specific interaction between EuS and $\mathrm{Au}$ in nanosystem under irradiation.

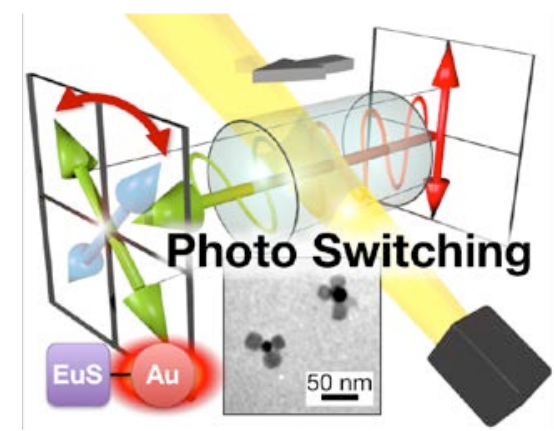

Illustration of photo-switch and TEM image of EuS-Au nanosystems.
1 Introduction Europium sulfide (EuS) is an intrinsic semiconductor with degenerate $4 f$ orbitals between the conduction band ( $5 d$ orbitals of $\mathrm{Eu}(\mathrm{II})$ ) and the valence band (3p orbitals of $\mathrm{S}^{2-}$ ) [1-3]. The $4 f-5 d$ electronic transition and spin configuration of EuS lead to a large magneto-optical effect, Faraday effect, which makes it a promising active material for future optical isolators for fiber-optic communication systems. In particular, synthesis and magneto-optical properties of EuS nanocrystals have been attracting attention for their optical, magnetic, and magneto-optical properties in visible region [4-12]. Recently, we have prepared EuS nanocrystals attached with gold ( $\mathrm{Au}$ ) nanoparticles using dithiol joint molecules
(EuS-Au nanosystems) [13]. The Au nanoparticle is known to exhibit localized surface plasmon resonance (LSPR) that leads to unique and unusual photophysical enhancements, such as enhanced luminescence and photo-catalytic properties [14-18]. We previously observed enhancement of the Faraday rotation angle and increase of the effective signals on the electron spin resonance (ESR) under visible light irradiation. The enhanced Faraday effects of EuS-Au nanosystems under visible light irradiation are expected to open up new optical devices using optical switching materials for fiber-optic communication systems.

In this study, photo-switching Faraday effects of EuS-Au nanosystems are demonstrated for the first time. 
The EuS nanocrystals and Au nanoparticles were synthesized by the thermal reduction of single-source precursors; tetrakis(diethyldithiocarbamate) tetraphenylphosphonium europium(III) $\left(\left(\mathrm{PPh}_{4}\right)\left[\mathrm{Eu}\left(\mathrm{S}_{2} \mathrm{CEt}_{2}\right)_{4}\right]\right)$ and chloro(triphenylphosphine) gold(I) $\left(\mathrm{PPh}_{3} \mathrm{AuCl}\right), \quad$ respectively. The EuS-Au nanosystems were prepared using ligand exchange reaction of 1,10-decanedithiol (Figure 1). The prepared EuS-Au nanosystems were characterized using transmission electron microscopy (TEM) and XRD measurements. The Faraday effects of toluene solution containing EuS-Au nanosystems were estimated using magnetic circular dichroism (MCD) measurements attached with visible light irradiation system (>440 nm). The photo-switchable Faraday effect is demonstrated, which might have applications in optical devices.
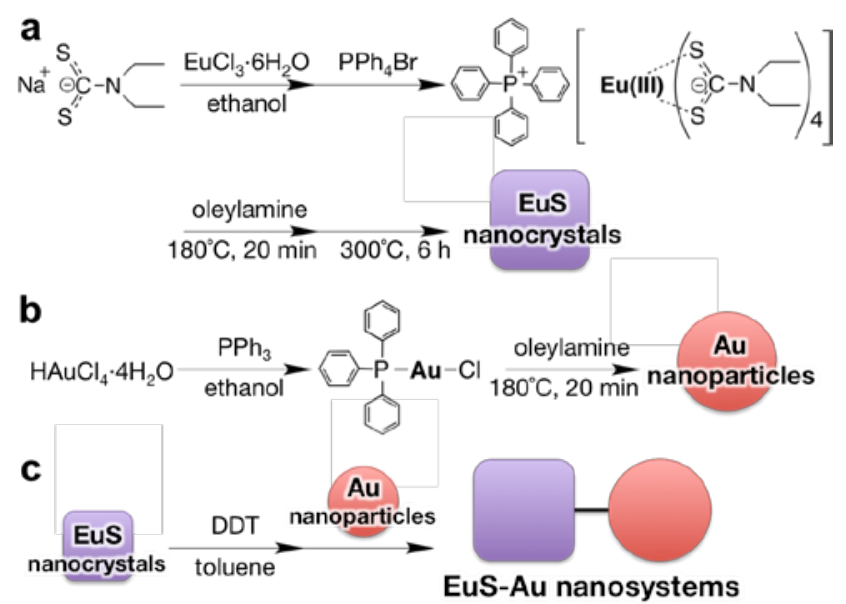

Figure 1 Reaction schemes of (a) cube-shaped EuS nanocrystals, (b) sphere-shaped Au nanoparticles and (c) EuS-Au nanosystems.

\section{Experiment}

2.1 Chemical reagents Europium(III) chloride hexahydrate $\left(\mathrm{EuCl}_{3} \cdot 6 \mathrm{H}_{2} \mathrm{O}\right)$ and sodium $\mathrm{N}, \mathrm{N}$-diethyldithiocarbamate trihydrate $\left(\mathrm{Na}\left(\mathrm{S}_{2} \mathrm{CNEt}_{2}\right) \cdot 3 \mathrm{H}_{2} \mathrm{O}\right)$ was purchased from Kanto Chemical Co. Inc. Tetrachloroaurate(III) tetrahydrate $\left(\mathrm{HAuCl}_{4} \cdot 4 \mathrm{H}_{2} \mathrm{O}\right)$, tetraphenylphosphonium bromide $\left(\mathrm{BrPPh}_{4}\right)$ and triphenylphosphine $\left(\mathrm{PPh}_{3}\right)$ were purchased from Wako Pure Chemical Industry, Ltd. Oleylamine and 1,10-decanedithiol (DDT) were obtained from Tokyo Chemical Industry Co., Ltd. All other chemicals and solvents were reagent grade and were used without further purification.

2.2 Apparatus Infrared spectra were recorded on a JASCO FT/IR-350 spectrometer. ${ }^{1} \mathrm{H}$ NMR data were measured by a JEOL AL-300 (300 MHz). ${ }^{1} \mathrm{H}$ NMR chemical shifts were determined by using tetramethylsilane (TMS) as an internal standard. Elemental analyses were performed with a Yanaco CHN corder MT-6. XRD patterns were characterized by a Rigaku SmartLab X-ray diffractometer. High-resolution images of the EuS-Au nanosystems were obtained with TEM, the JEOL 2010 FASTEM (200 kV). UV-vis absorption spectra were measured on a JASCO V-570 spectrophotometer at room temperature.

2.3 Synthesis of Tetraphenylphosphonum Tetrakis(diethyldithiocarbamate) Europium(III) $\left(\left(\mathrm{PPh}_{4}\right)\left[\mathrm{Eu}\left(\mathrm{S}_{\mathbf{2}} \mathrm{CEt}_{2}\right)_{4}\right]\right) \quad \mathrm{A}$ solution of $\left(\mathrm{Na}\left(\mathrm{S}_{2} \mathrm{CNEt}_{2}\right) \cdot 3 \mathrm{H}_{2} \mathrm{O}\right)(9.00 \mathrm{~g}, 39.9 \mathrm{mmol})$ in methanol (32 $\mathrm{mL})$ was added to $\mathrm{EuCl}_{3} \cdot 6 \mathrm{H}_{2} \mathrm{O}(2.80 \mathrm{~g}, 7.64 \mathrm{mmol})$ dissolved in methanol (32 $\mathrm{mL})$ while stirring and reacted for $3 \mathrm{~h}$. After the reaction mixture was filtered, a solution of $\mathrm{BrPPh}_{4}$ (16.04 g, $\left.38.26 \mathrm{mmol}\right)$ in methanol $(20 \mathrm{~mL})$ was added to the filtrated solution and stirred for $1 \mathrm{~h}$. The resulting precipitate was separated by filtration and washed 2 times with ethanol. ${ }^{1} \mathrm{H}$ NMR (300 $\mathrm{MHz}, \mathrm{CDCl}_{3}, 25^{\circ} \mathrm{C}$, TMS): $\delta=7.90-7.60$ (m, 20H; Ar-H), 2.80-2.65 (m, 16H; $\left.\mathrm{CH}_{2}\right), 1.61 \mathrm{ppm}\left(\mathrm{t},{ }^{3} \mathrm{~J}(\mathrm{H}, \mathrm{H})=7 \mathrm{~Hz}, 24 \mathrm{H} ; \mathrm{CH}_{3}\right)$; IR $(\mathrm{KBr})$ : $\tilde{v}=1485-1482 \mathrm{~cm}^{-1}(\mathrm{C}-\mathrm{N}), 1442 \mathrm{~cm}^{-1}$ (P-Phenyl), 1007 $\mathrm{cm}^{-1}$ (C-S); elemental analysis calcd (\%) for $\mathrm{C}_{44} \mathrm{H}_{60} \mathrm{EuN}_{4} \mathrm{PS}_{8}$ : C 48.73, H 5.58, N 5.17, S 23.65; found: C 48.55, H 5.51, N 5.00, S 23.41.

2.4 Synthesis of Cube-Shaped EuS Nanocrystals Under $\mathrm{N}_{2}$ atmosphere $\left(\mathrm{PPh}_{4}\right)\left[\mathrm{Eu}\left(\mathrm{S}_{2} \mathrm{CNEt}_{2}\right)_{4}\right]$ (1.91 g, $\left.1.76 \mathrm{mmol}\right)$ was dissolved into oleylamine $(10 \mathrm{~mL}, 30.4 \mathrm{mmol})$, and the mixture was heated at $180{ }^{\circ} \mathrm{C}$ and stirred for $20 \mathrm{~min}$. After the reaction solvent was heated to $300{ }^{\circ} \mathrm{C}$ and stirred for $6 \mathrm{~h}$, the purple liquid was centrifuged at $4000 \mathrm{rpm}$ for $10 \mathrm{~min}$. The precipitation was added to toluene $(10 \mathrm{~mL})$ and centrifuged at $4000 \mathrm{rpm}$ for $15 \mathrm{~min}$, and the clear purple liquid of oleylamine capped EuS nanocrystals was obtained.

2.5 Synthesis of Chloro(triphenylphosphine) Gold(I) (PPh $\mathbf{3}$ AuCl) A solution of $\mathrm{HAuCl}_{4}(10.0 \mathrm{~g}, 24.3$ $\mathrm{mmol})$ in ethanol $(150 \mathrm{~mL})$ was added, with stirring, to $\mathrm{PPh}_{3}$ (12.7 g, $\left.48.6 \mathrm{mmol}\right)$ dissolved in ethanol (100 mL) heated under reflux for several hours, and cooled to room temperature. The resulting precipitate was separated by filtration and washed 2 times with ethanol. Recrystallization from hexane gave white crystals of $\mathrm{PPh}_{3} \mathrm{AuCl} .{ }^{1} \mathrm{H}$ NMR (300 MHz, $\mathrm{CDCl}_{3}, 25^{\circ} \mathrm{C}, \mathrm{TMS}$ ): $\delta$ $=7.50 \mathrm{ppm}$ (m, 15H, Ar-H); IR (KBr): $\tilde{v}=1407 \mathrm{~cm}^{-1}(\mathrm{P}-$ Phenyl); elemental analysis calcd (\%) for $\mathrm{C}_{18} \mathrm{H}_{15} \mathrm{AuCl}$ : C 43.70, H 3.06, N 0; found: C 43.58, H 3.02, N 0.

2.6 Synthesis of Au Nanoparticles Under $\mathrm{N}_{2}$ atmosphere $\mathrm{PPh}_{3} \mathrm{AuCl}(0.50 \mathrm{~g}, 1.01 \mathrm{mmol})$ was dissolved into oleylamine (30 mL, $91.2 \mathrm{mmol}$ ), and the mixture was heated at $180{ }^{\circ} \mathrm{C}$ and stirred for $30 \mathrm{~min}$. After the solution was cooled to room temperature, the red liquid was centrifuged at $4000 \mathrm{rpm}$ for $10 \mathrm{~min}$. The precipitation was added to THF (20 mL) and centrifuged at $4000 \mathrm{rpm}$ for 15 $\mathrm{min}$, and the clear red liquid of oleylamine capped $\mathrm{Au}$ nanoparticles was obtained.

2.7 Ligand Exchange Reactions Prepared EuS nanocrystals suspended in toluene solution $(20 \mathrm{ml})$ with 
EuS nanocrystals $(0.08 \mathrm{~g})$ is added into toluene $(5 \mathrm{~mL})$ with DDT $(0.1 \mathrm{~mL})$. After $5 \mathrm{~min}$, the toluene solution including EuS-DDT was added into excess amount of 1-dodecanethiol, and the liquid of dithiol capped EuS nanocrystals (EuS-DDT) was obtained.

2.8 Synthesis of EuS-Au Nanosystems The EuS-Au nanosystems were formed by adding the solution of $\mathrm{Au}$ nanoparticles $(0.05 \mathrm{~g})$ in THF $(20 \mathrm{~mL})$ to the solution of EuS-DDT in toluene. The mixture was stirred for $30 \mathrm{~min}$ at room temperature. The solution was added to 1-dodecanethiol (1 mL) and stirred for $1 \mathrm{~h}$. The resulting solution of EuS-Au nanosystems in toluene was obtained.

2.9 MCD measurements MCD measurements were performed using a JASCO J-1500 spectrodichrometer equipped with a JASCO permanent magnet $(0.47 \mathrm{~T})$. For MCD measurements under light irradiation, a metal halide lamp (> $440 \mathrm{~nm}$, Moritex, MHF100L II) was employed as the excitation light source. The schematic view of experimental setup is shown in Figure 2.

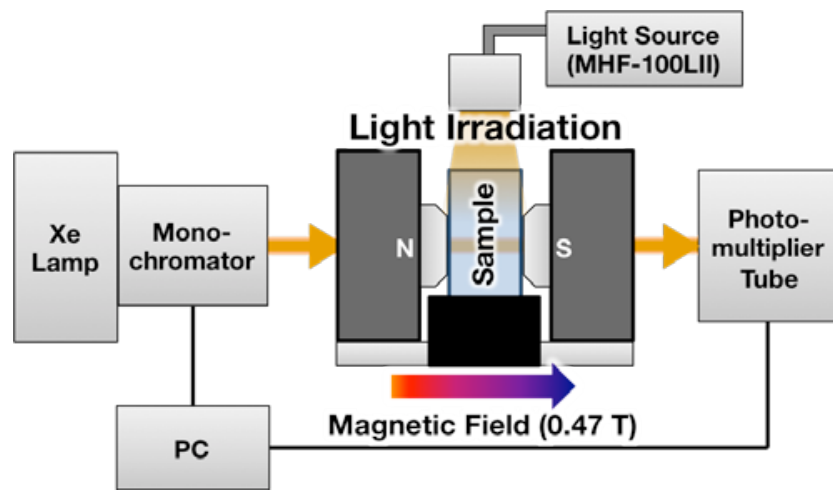

Figure 2 Schematic view of experimental setup for MCD measurements under light irradiation.

3 Results and Discussion Monodisperse EuS nanocrystals and Au nanoparticles were synthesized by thermal reaction of $\mathrm{Eu}(\mathrm{III})$ dithiocarbamate complex $\left(\left(\mathrm{PPh}_{4}\right)\left[\mathrm{Eu}\left(\mathrm{S}_{2} \mathrm{CNEt}_{2}\right)_{4}\right]\right)$ and $\mathrm{Au}(\mathrm{I})$ triphenyl complex $\left(\mathrm{PPh}_{3} \mathrm{AuCl}\right)$ as single source precursors in oleylamine, respectively (Figure 1 and see supporting information Table S1). Prepared EuS nanocrystals were connected with $\mathrm{Au}$ nanoparticles using dithiol joint molecules (DDT), resulting in formation of EuS-Au nanosystems. The XRD patterns of EuS nanocrystals, Au nanoparticles, and EuS-Au nanosystems are shown in Figure 3. The diffraction peaks at $2 \theta=25.8,29.9,42.8,50.6,53.1,62.2$, 68.5 , and 70.5 degree were assigned to the (111), (200), (220), (311), (222), (400), (331), and (420) planes of $\mathrm{NaCl}$ type EuS (ICDD 26-1419). The diffraction peaks at $2 \theta=$ 38.2, 44.4, 64.7, and 78.5 degree were also assigned to the (111), (200), (220), and (311) planes of face-centered-cubic (fcc) type Au (ICDD 4-0784). We evaluated the full-width at half maximum (FWHM) of the $2 \theta=25.8$ and 38.2 degree peaks, which correspond to Bragg diffraction from the EuS (200) and Au (111) planes, respectively. The FWHM of EuS and Au nanocrystals in EuS-Au nanosystems were found to be 0.49 and 0.50 degree, respectively, although the FWHM of the previous EuS and Au nanocrystals were found to be 0.58 and 0.67 degree, respectively. A smaller EuS-Au nanosystems might be removed by centrifugation process for purification of EuS-Au nanosystems. The EuS and $\mathrm{Au}$ crystallite sizes calculated from the Scherer equation from the XRD data were found to be 16.6 and $16.8 \mathrm{~nm}$, respectively.

We observed cube-shaped EuS nanocrystals and sphere-shaped Au nanoparticles using the transmission electron microscopy (TEM) (Figure $4 \mathrm{a}$ and b). We estimated that the average crystal grain sizes of EuS and Au nanocrystals were found to be 19.5 and $18.5 \mathrm{~nm}$ from TEM images, respectively. The TEM image of EuS-Au nanosystems is shown in Figure 4c. The TEM image indicates the formation of EuS-Au nanosystems composed of cube-shaped EuS nanocrystals and spherical Au nanoparticles. We also observed very few EuS nanocrystals without $\mathrm{Au}$ nanoparticles (see supporting information Figure S1). Spherical Au nanoparticles (black circles) was surrounded with cubic EuS nanocrystals. The sizes of EuS nanocrystals (approximately $19.5 \mathrm{~nm}$ ) and Au nanoparticles (approximately $18.5 \mathrm{~nm}$ ) were similar to that of estimation values from XRD measurements.

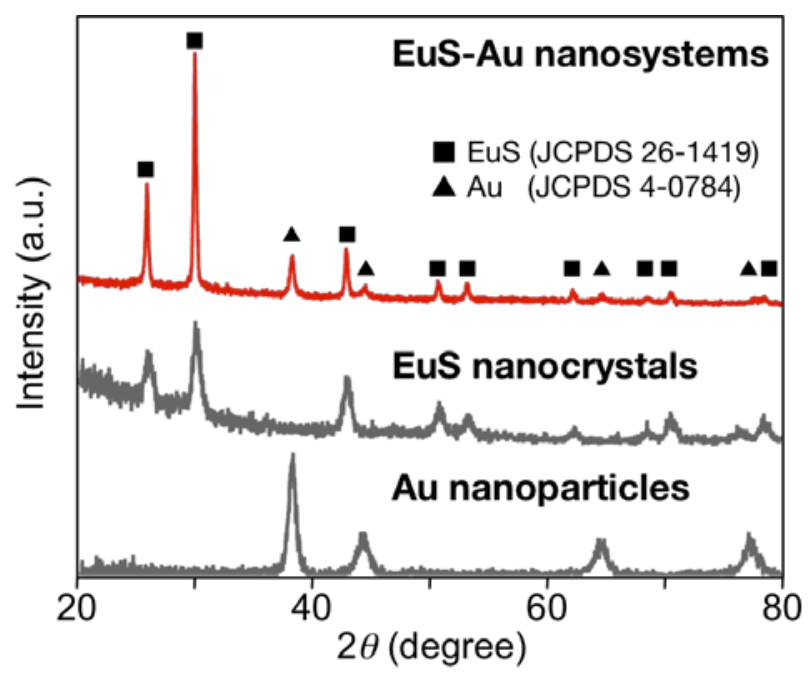

Figure 3 XRD patterns of EuS-Au nanosystems, EuS nanocrystals, and Au nanoparticles. 

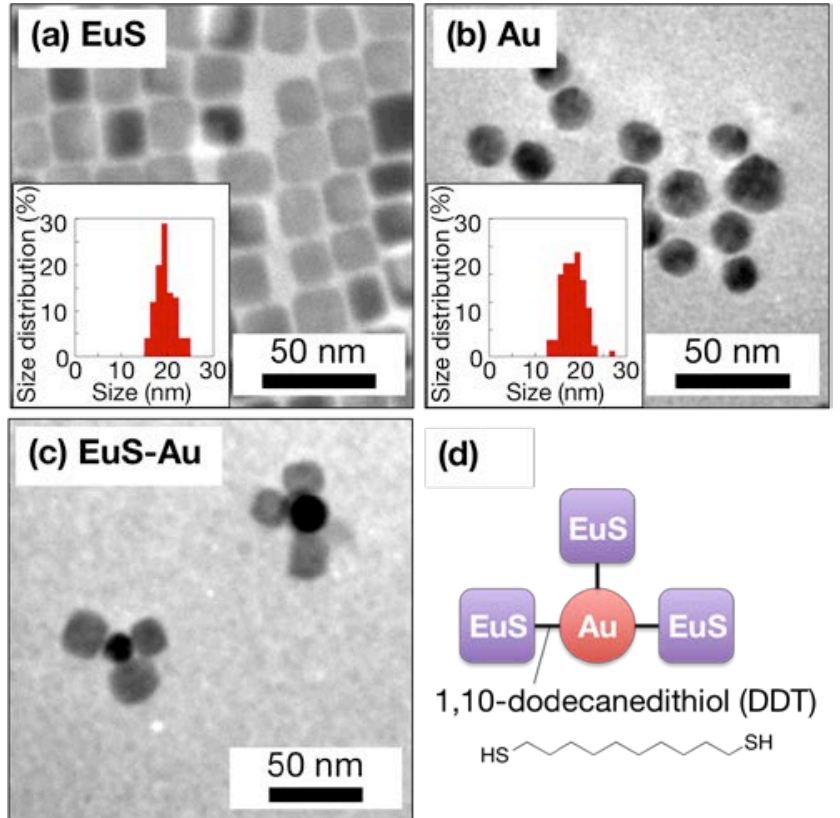

(d)

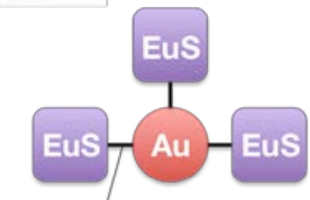

1,10-dodecanedithiol (DDT)

HSH

Figure 4 TEM images of (a) EuS nanocrystals, (b) $\mathrm{Au}$ nanoparticles, and (c) EuS-Au nanosystems. (d) Illustration of the EuS-Au nanosystems linked with 1,10-decanedithiol (DDT). Inset of Fig. 4a and b show the size distributions of EuS and Au nanocrystals, respectively.

Prepared EuS-Au nanosystems are dispersed in toluene, homogeneously. The absorption spectra of toluene solution containing EuS nanocrystals and EuS-Au nanosystems and of THF solution containing Au nanoparticles are shown in Figure 5a and b, respectively. The broad absorption band at $510 \mathrm{~nm}$ is assigned to the $4 f-5 d$ transition in the EuS nanocrystals. The broadening of absorbance of EuS-Au nanosystems at around $600 \mathrm{~nm}$ was observed, although the LSPR band of only Au nanoparticles is shown at around $535 \mathrm{~nm}$ (Figure 5b). The broad absorbance band of EuS-Au nanosystems might be caused by dielectric surrounding environments around $\mathrm{Au}$ nanoparticles [19-21]. The red shift of the LSPR band means that Au nanoparticles directly linked to EuS nanocrystals with large dielectric environment. From these results, we successfully formed EuS-Au nanosystems linked by DDT. Additionally, the EuS nanocrystals have potential to be affected by the LSPR of Au nanoparticles between 530 to $800 \mathrm{~nm}$.

Under visible light irradiation, we observed drastic change of absorption band of EuS-Au nanosystems for the first time (Figure 5b). The absorption intensity change under visible light and dark at around $600 \mathrm{~nm}$ can be repeated for many times, reversibly. On the other hand, the drastic change of absorption band in toluene solution with EuS nanocrystals was not observed. The drastic absorption band change of EuS-Au nanosystems at around $600 \mathrm{~nm}$ might be caused by enhancement of LSPR and decreasing the ground state density of EuS-Au nanosystems under irradiation.
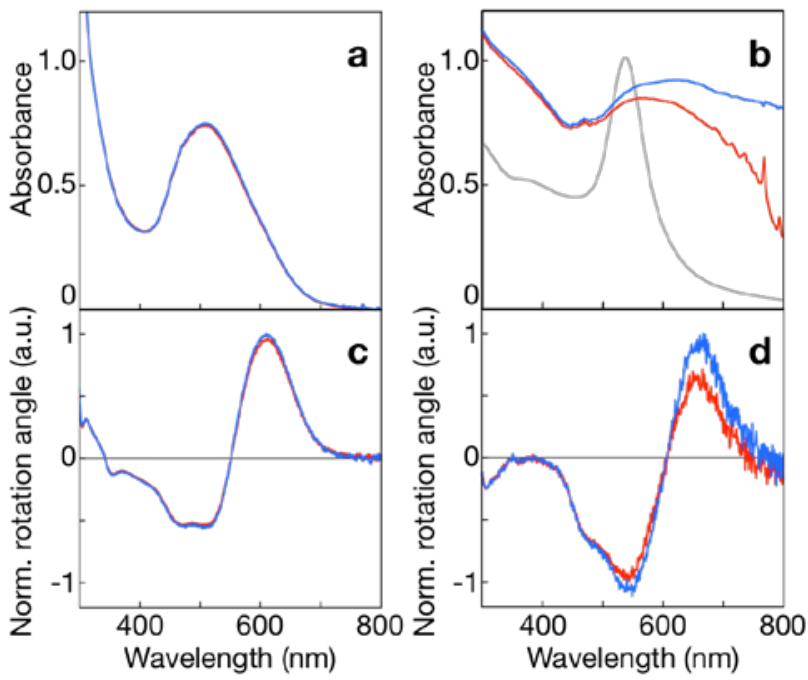

Figure 5 UV-Vis absorption spectra and MCD spectra of toluene solution containing EuS nanocrystals (a and c) and EuS-Au nanosystems (b and d) under dark (blue line) and light irradiation (red line) with an external magnetic field of $0.47 \mathrm{~T}$. Gray line in Fig. 5b is UV-Vis absorption spectrum of THF solution containing Au nanoparticles.

The MCD spectra of EuS nanocrystals and EuS-Au nanosystems under irradiation and dark are shown Figure 5c and $d$. In order to estimate the ratio between MCD spectra under dark and light irradiation, we consider that the MCD spectra should be normalized by the peak values of dark condition. The EuS-Au nanosystems exhibit positive and negative rotation signal as well as the EuS nanocrystals. This indicates that they might be caused by the excited state degeneracy of Eu ion, which is assigned to the $4 f-5 d$ transition in the EuS nanocrystals. The rotation wavelengths of EuS-Au nanosystems (positive and negative rotation signal at around 670 and $550 \mathrm{~nm}$, respectively) are longer than those of EuS (610 and 510 $\mathrm{nm})$. Additionally, the ratio of negative rotation angle to positive of EuS-Au nanosystems (1.04) is about twice larger than that of EuS nanocrystals (0.56). These results indicate that the Au nanoparticles affect the excited and grand state of EuS nanocrystals and then MCD spectrum is changed [22]. Under irradiation, effective changes of MCD spectrum of EuS-Au nanosystems in toluene solution were observed at around 670 and $550 \mathrm{~nm}$ (maximum positive and negative rotation wavelength), although the MCD spectrum of EuS nanocrystals in toluene solution shows slight change at around $610 \mathrm{~nm}$. The effective reversible changes in MCD spectra at $670 \mathrm{~nm}$ with the alternative irradiation cycles of visible light $(>440 \mathrm{~nm}$ ) and dark are also shown in Figure 6. From these alternative irradiation experiments, reversible change of rotation angles of EuS-Au nanosystems may not be caused by aggregation or decomposition of EuS-Au nanosystems. In addition, we estimated the decrease rate of rotation angle of EuS-Au nanosystems using Equation (1). 


$$
\Delta \theta=\left(\theta_{\text {dark }} / A_{\text {dark }}-\theta_{\text {light }} / A_{\text {light }}\right) /\left(\theta_{\text {dark }} / A_{\text {dark }}\right)
$$

where $\theta$ and $A$ are the rotation angle of MCD spectra and absorbance at maximum positive rotation wavelength $(670 \mathrm{~nm})$, respectively. In order to estimate the Faraday rotation without effect on the absorption band, we calculated the decrease rate using equation (1). The decrease rate $(\Delta \theta)$ of EuS-Au nanosystems was found to be $38.0 \%$ although the relation between decrease rates of absorbance and MCD intensity might be linear [22]. This result indicates that the decrease rate of rotation angle at $670 \mathrm{~nm}$ of EuS-Au nanosystems is larger than that of absorbance. We consider that the effective change of MCD spectra of EuS-Au nanosystems would be dominated not only by drastic change of absorption band related to enhanced LSPR of Au nanoparticles but also by specific interaction between $\mathrm{EuS}$ and $\mathrm{Au}$ in nanosystem under irradiation. There are mainly two specific interactions between EuS nanocrystals and $\mathrm{Au}$ nanoparticles, the mixing of electronic state between EuS and $\mathrm{Au}$ nanocrystals and the influence of LSPR on EuS nanocrystals. In particular, LSPR of $\mathrm{Au}$ nanoparticles might cause the change in absorption of EuS nanocrystals. Although it is difficult to determine the exact origin of the phenomena, the LSPR is one of the origins inducing the weak MCD intensity [22].

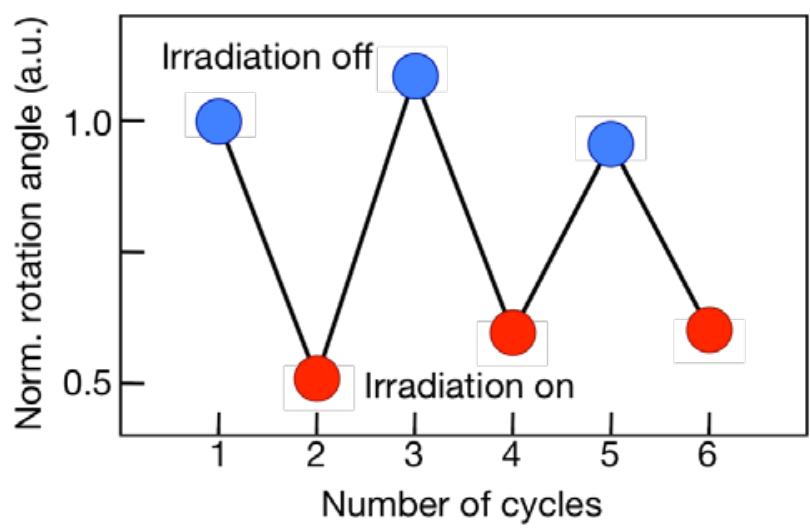

Figure 6 The reversible changes in MCD spectra of EuS-Au nanosystems at $670 \mathrm{~nm}$ with the alternative irradiation cycles of visible light (> $440 \mathrm{~nm})$ and dark.

4 Conclusions Novel photo-switching Faraday effects of EuS-Au nanosystems are successfully observed for the first time. The drastic change of absorption band may be related to enhanced LSPR and specific interaction EuS and $\mathrm{Au}$ in nanosystems under irradiation. We are now trying to fabricate an optical switching system using polymer materials including EuS-Au nanosystems. The Photo-switchable EuS-Au nanosystems with Faraday effects are expected to be novel photo-switching material for advanced fiber-optic communication systems.
Acknowledgements This work was partly supported by the Ministry of Education, Culture, Sports, Science and Tech (MEXT), Japan through Grants-in-Aid for Scientific Research on Innovative Areas of "Emergent chemistry of nanoscale molecular systems" and "New Polymeric Materials Based on Element-Blocks (No.2401)” (24102012), Grand-in-Aid for Young Scientists (B) (15K18206), and Grant-in-Aid for JSPS Fellows (26.1186). We also thank for the support of Frontier Chemistry Center Akira Suzuki "Laboratories for Future Creation” Project.

\section{References}

[1] P. Wachter, CRC Crit. Rev. Solid State Sci. 3, 189 (1972).

[2] T. Kasuya, J. Magn. Magn. Mater. 195, 141 (1999).

[3] J. Kunes, W. Ku, and W. E. Pickett, J. Phys. Soc. Jpn. 74, 1408 (2005).

[4] J. C. Suits, B. E. Argyle, and M. J. Freiser, J. Appl. Phys. 37, 1391 (1966).

[5] T. Mirkovic, M. A. Hines, P. S. Nair, and G. D. Scholes, Chem. Mater. 17, 3451 (2005).

[6] T. Kataoka, Y. Tsukahara, Y. Hasegawa, and Y. Wada, Chem. Commun. 6038 (2005).

[7] Y. Hasegawa, M. Afzaal, P. O'Brien, Y. Wada, and S. Yanagida, Chem. Commun. 0, 242 (2005).

[8] R. S. Selinsky, J. H. Han, E. A. Morales Pérez, I. A. Guzei, and S. Jin, J. Am. Chem. Soc. 132, 15997 (2010).

[9] A. Tanaka, H. Kamikubo, M. Kataoka, Y. Hasegawa, and T. Kawai, Langmuir 27, 104 (2010).

[10] W. L. Boncher, E. A. Görlich, K. Tomala, J. L. Bitter, and S. L. Stoll, Chem. Mater. 24, 4390 (2012).

[11] Y. Hasegawa, M. Kumagai, A. Kawashima, T. Nakanishi, K. Fujita, K. Tanaka, and K. Fushimi, J. Phys. Chem. C 116, 19590 (2012).

[12] Y. Hasegawa, M. Maeda, T. Nakanishi, Y. Doi, Y. Hinatsu, K. Fujita, K. Tanaka, H. Koizumi, and K. Fushimi, J. Am. Chem. Soc. 135, 2659 (2013).

[13] A. Kawashima, T. Nakanishi, T. Shibayama, S. Watanabe, K. Fujita, K. Tanaka, H. Koizumi, K. Fushimi, and Y. Hasegawa, Chem. Eur. J. 19, 14438 (2013).

[14] O. Kulakovich, N. Strekal, A. Yaroshevich, S. Maskevich, S. Gaponenko, I. Nabiev, U. Woggon, and M. Artemyev, Nano Lett. 2, 1449 (2002).

[15] E. Katz, and I. Willner, Angew. Chem. Int. Ed. 43, 6042 (2004).

[16] J. Lee, A. O. Govorov, J. Dulka, and N. A. Kotov, Nano Lett. 4, 2323 (2004).

[17] Y. Tian, and T. Tatsuma, J. Am. Chem. Soc. 127, 7632 (2005).

[18] A. V. Zayats, I. I. Smolyaninov, and A. A. Maradudin, Phys. Rep. 408, 131 (2005).

[19] B. N. J. Persson, Surf. Sci. 281, 153 (1993).

[20] J. R. Lakowicz, Anal. Biochem. 337, 171 (2005). 
[21] V. Lesnyak, A. Wolf, A. Dubavik, L. Borchardt, S. V. Voitekhovich, N. Gaponik, S. Kaskel, and A. Eychmuller, J. Am. Chem. Soc. 133, 13413 (2011).

[22] J. Larrabee, G. Schenk, N. Mitić, and M. Riley, Eur. Biophys. J. 44, 393 (2015). 